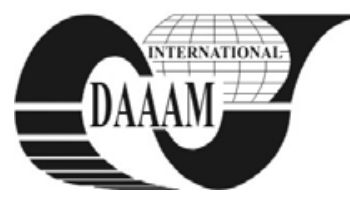

Annals of DAAAM for 2011 \& Proceedings of the 22nd International DAAAM Symposium, Volume 22, No. 1, ISSN $1726-9679$ ISBN 978-3-901509-83-4, Editor B. Katalinic, Published by DAAAM International, Vienna, Austria, EU, 2011 Make Harmony between Technology and Nature, and Your Mind will Fly Free as a Bird

\title{
FEATURES OF EXPERIMENTAL RESEARCH OF METHOD OF TELECONTROL BY MANIPULATOR
}

\author{
KATSURIN, A[lexey] \& FILARETOV, V[ladimir] F[edorovich]
}

\begin{abstract}
This paper describes the features of experimental research of method of semiautomatic position telecontrol by a manipulator using a special setting device (SD). The television camera in zone of work can change its spatial orientation. It can rotate around two mutually perpendicular axes by operator commands. The results of executed experiments confirm effectiveness of this method

Key words: telecontrol, manipulator, mobile telecamera
\end{abstract}

\section{INTRODUCTION}

The regime of semiautomatic control is frequently used for rapid and precise execution of some critical operations in the extreme cases. The human-operator sets the motion of manipulator grip or any other operating tool by means of the setting device or control handle and looks only on the image of working space on the screen of monitor. Projects in the area of telecontrol by various robots and manipulators are realized by many researchers in different countries (Changhwan et al., 2006; Tarca et al., 2007). However many key problems are not still solved. Often several telecameras must be located in the zone of works with different orientation of their optical axes or the used telecamera must have capability to move in space and to change its orientation. In this case the operator must constantly take into account mutual orientation of SD, manipulator and telecamera in the process of the setting of grip motions. It leads to the large load on the man.

The approach, which makes it possible to consider the current orientation of the optical axis of mobile telecamera, is proposed in the paper (Murao et al., 2005). But the task of the formation of required control signals for manipulator with using SD is not examined when this orientation is changed. The work (Filaretov et al., 2001) resolves the problem of telecontrol by manipulator when the telecamera orientation changes. But the questions of initial co-ordination of the image of manipulator on the monitor screen and the real position of SD are not examined. Furthermore in this work it is not solved the problem of the elimination of ambiguities which appear during calculation of some trigonometric functions when the inverse task of kinematics is solved. The paper (Filaretov \& Katsurin, 2008) resolves the problem of telecontrol by multi-link manipulator when the telecamera orientation changes. But paper includes only mathematical simulation of work of telecontrol system without experimental researches.

As a result the problem of experimental researches of method and algorithm of semiautomatic telecontrol by manipulators with a changing of orientation the optical axis of television camera, which is located in the zone of the realization of working operations, is remained the important.

\section{FORMULATION OF THE PROBLEM}

This paper resolves the problem of experimental researches of the method of semiautomatic position telecontrol by manipulator using the special SD. In this case it is necessary to receive control laws (setting signals for manipulator drives) and to take into account current orientation of a television camera, which is established in the work zone and formed image on the operator monitor in the process of realization of work operations. This camera can change orientation of its optical axis per rotating around two mutually perpendicular axes. The operator can choose its orientations for reception of the best view of working zone.

\section{SYSTEM OF TELECONTROL BY THE MANIPULATOR}

Real robotic equipment which used for experimental researches has kinematic schemes differing from schemes in paper (Filaretov \& Katsurin, 2008). Figure 1 shows the scheme of the semiautomatic telecontrol system by the spatial motion of manipulator 4 using the special setting device 1 . Figure 1 has following designations: $C_{i} \quad(i=\overline{1,5})$ - the systems of coordinates (SC) rigidly connected, correspondingly, with the basis of SD, the basis of SD handle, the basis of manipulator, the characteristic point of manipulator and the body of television camera 3; $q_{i}(i=\overline{1,8})$ - the generalized coordinates of corresponding degrees of freedom of $\mathrm{SD}$, manipulator and television camera; 2 - monitor.

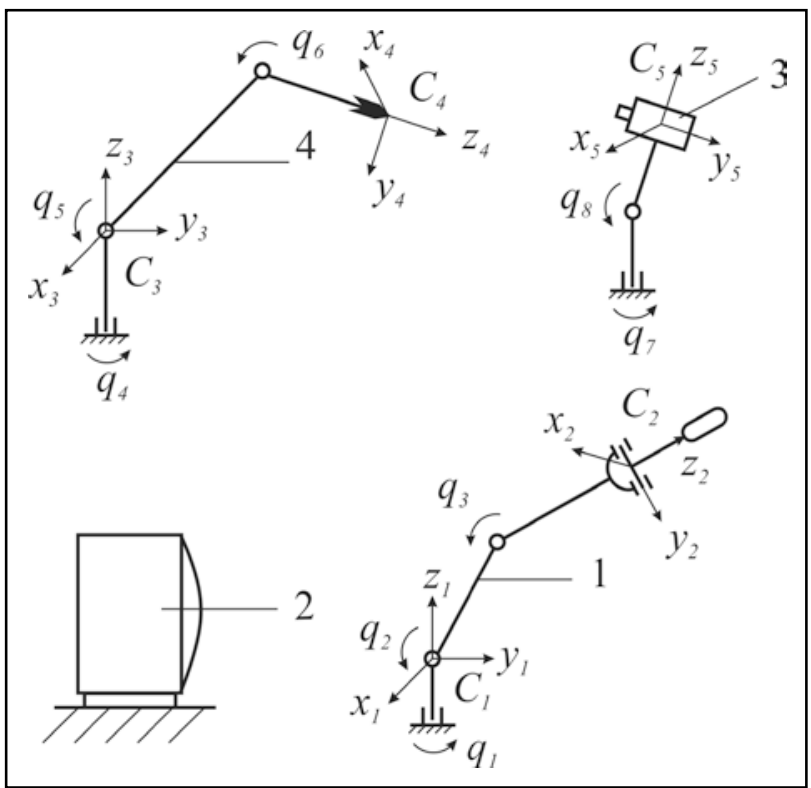

Fig. 1. The scheme of the system of telecontrol by manipulator

The equipment PHANTOM Omni and Premium 1.5 (SensAble Technologies, Inc.) have been used as setting devices and manipulator. It was used two setting devices: first for control of the manipulator, second for change of orientation of a television camera (Fig. 2). The location of the mobile camera and two identical manipulators (during the experiments 
one of them has been switched off) is shown on Fig. 3. The mobile camera is established on the robot miniCRANE 5.1 (Robots and Design, Co. Ltd.), which provide turn of the camera around two perpendicular axes.

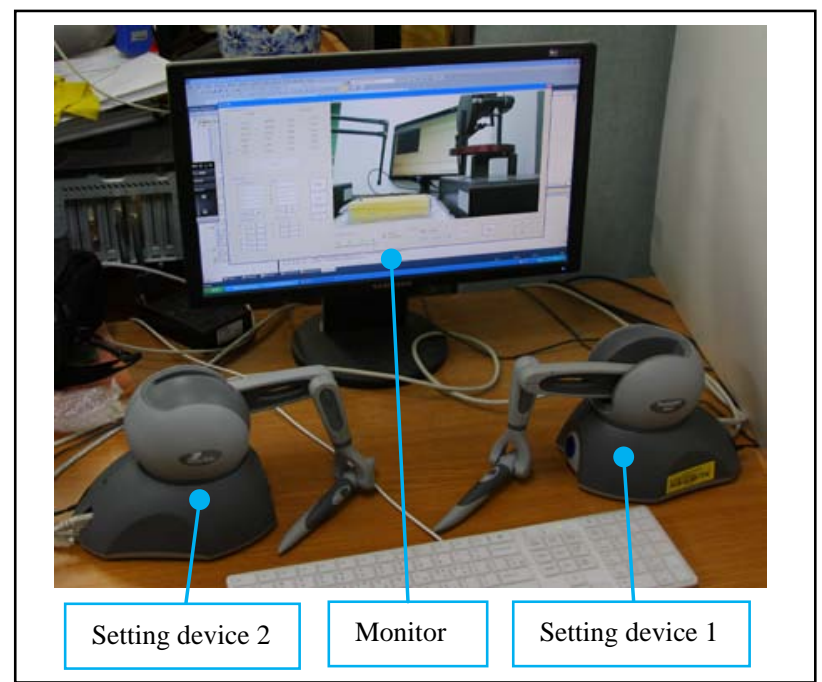

Fig. 2. Operator place

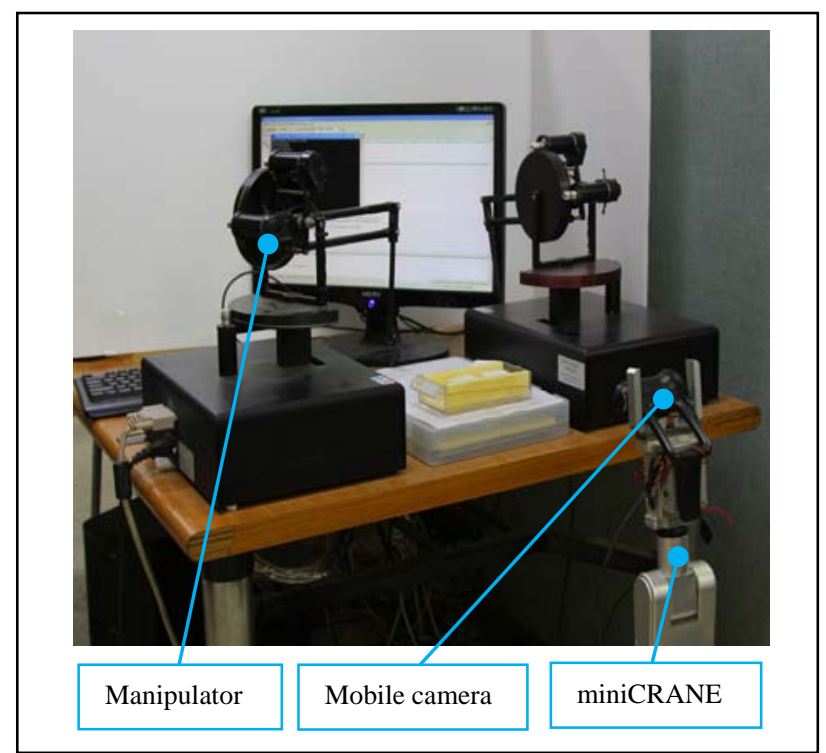

Fig. 3. Working space

The system of position telecontrol by the manipulator (see Fig.1-3) has two regimes (Filaretov et al., 2001; Filaretov \& Katsurin, 2008): the regime of stabilization and the regime of tracking. The first regime is auxiliary and the second is basic. In the regime of stabilization the manipulator is fixed in the space and the operator has the capability to change SD configuration for more comfortable executing of subsequent operations. In the same regime operator can change an orientation of optical axis of telecamera in the work zone.

At switching moment from the regime of stabilization to the regime of tracking the setting signals for drives of all degrees of freedom of manipulator are formed. These signals provide the co-ordination of its position with the SD orientation and take into account the current orientation of the telecamera optical axis. Subsequently the manipulator repeats any motions formed by SD in the regime of tracking.

Using this system the operator always has the opportunity to switch from the regime of tracking to the regime of stabilization, to study the current situation, to select the most rational new configuration of SD and to continue execution of technological operations again in the regime of tracking. For the realization of this method of semiautomatic telecontrol by manipulator the algorithm of work of the computing system which ensures the formation of setting signals for drives of all degrees of freedom of manipulator was developed too.

\section{EXPERIMENT}

Experiments with real robotic equipment (see Fig. 2-3) for confirmation capacity for work and effectiveness of system of position telecontrol by manipulator have been made. On Fig. 4 the experimental results of system work with the turned telecamera are shown. On Fig. 4 the line 1 coincides with a trajectory of motion of SD handle in SC $C_{1}$. The line 2 coincides with direction of SD motion in SC $C_{3}$, i.e. line 2 assigns the direction of manipulator motion in $\mathrm{SC} C_{3}$. The line 3 shows a trajectory of motion of characteristic point of manipulator grip in SC $C_{3}$. Consequently the manipulator on the screen of the operator monitor moved strictly in the direction, which is set using the SD at any orientation of the camera.

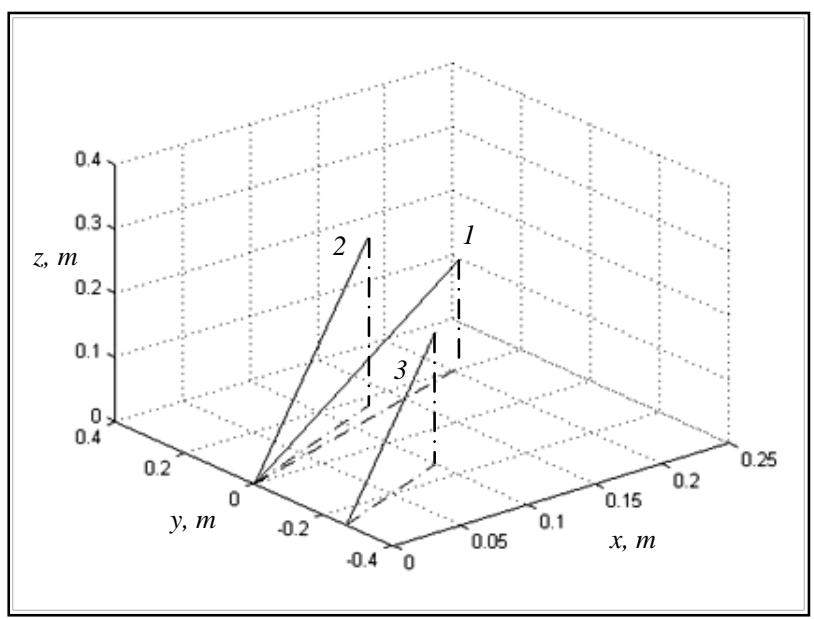

Fig. 4. Motion of SD and characteristic point of manipulator

\section{CONCLUSION}

Results of the executed experiments confirm capacity for work and effectiveness of the method of semiautomatic position telecontrol by the manipulator. This method makes it possible to carry out complex technological operations using the elementary SD and to automatically take into account the spatial orientation of television camera. Also in this case the psychological load on human-operator is reduced.

\section{REFERENCES}

Changhwan, C.; Yongchil, S.; Seungho, J.; Seungho, K. (2006). Tele-Operated Fuel Handling Machine Manipulation Robot for the Nuclear Power Plants, Proceedings of the 6-th Asian Control Conf., Bali, Indonesia, pp. 983-987.

Filaretov, V.; Alekseev, Yu.; Lebedev A. (2001). Control systems of underwater robots, Krugly god, Moscow.

Filaretov, V.; Katsurin, A. (2008). Method of semiautomatic position control by manipulator using telecamera which changes spatial orientation of its optical axis. Mechatronics, automation, control, No. 9, pp. 15-22.

Murao, T.; Kawai, H.; Fujita, M. (2005). Passivity-based dynamic visual feedback control with a movable camera, Proceedings of 16 IFAC World Congress, Prague, Czech Rep., pp. 1-6.

Tarca, R.; Pasc, I.; Tarca, N.; Popentiu-Vladicescu F. (2007). Remote robot control Via Internet Using Augmented Reality, Proceedings of the 18-th DAAAM Int. Symp. 